\title{
Quantum logic gates using Stark-shifted Raman transitions in a cavity
}

\author{
Asoka Biswas and G. S. Agarwal \\ Physical Research Laboratory, Navrangpura, Ahmedabad 380 009, India
}

(Received 18 January 2004; published 4 June 2004)

\begin{abstract}
We present a scheme to realize the basic two-qubit logic gates such as the quantum phase gate and the controlled-NOT gate using a detuned optical cavity interacting with a three-level Raman system. We discuss the role of Stark shifts, which are as important as the terms leading to the two-photon transition. The operation of the proposed logic gates involves metastable states of the atom and hence is not affected by spontaneous emission. These ideas can be extended to produce multiparticle entanglement.
\end{abstract}

DOI: 10.1103/PhysRevA.69.062306

PACS number(s): 03.67.Lx

\section{INTRODUCTION}

The performance of a quantum computer relies on certain universal one-qubit and two-qubit logic gates. Any quantum computation [1] can be reduced to a sequence of these gates $[2,3]$. There have been a number of experimental systems proposed as candidates for implementing these logic gates, and many of these have been implemented. We may mention trapped ions [4], cavity quantum electrodynamics [5-7], nuclear magnetic resonance [8,9], quantum dots $[9,10]$, and neutral atoms in an optical lattice [11] as examples. Some of the basic two-qubit logic gates are the conditional quantum phase gate (QPG) [12], the controlled-NOT (CNOT) gate, which is a universal two-qubit gate $[3,9]$, the SWAP gate, etc. It should be mentioned that a $\pi$ shift of QPG and appropriate rotation of the second qubit realize the CNOT gate.

The QPG operation can be performed using a three-level atom interacting with a detuned cavity. The two-photon transitions are especially attractive in this case as then one can work with long-lived ground states of the atom. In such a situation, the excited state does not participate in the transition and thus it is possible to minimize the effect of decoherence associated with the finite lifetime of the excited state $[13,14]$. However, the two-photon transitions have complications associated with Stark shifts of the energy levels. The Stark shifts are quite natural to any two-photon process as one considers single-photon transitions which are detuned from the intermediate levels. If one ignores Stark shifts, as is very often done, then the nature of the two-photon process becomes similar to the one-photon process and many of the results such as Rabi oscillations carry over to two-photon processes. In this paper, we consider a situation where a three-level atom in the $\Lambda$ configuration interacts with a bimodal cavity where the modes are highly detuned from the corresponding one-photon transition. We demonstrate the possibility of performing a number of logic operations (e.g., QPG, CNOT, and SWAP) using the two-photon Raman transition. We show this in spite of the nonzero Stark shifts in the Raman transitions.

The structure of the paper is as follows. In Sec. II, we present the model system and its theoretical description. In Sec. III, we show how different two-qubit logic gate operations can be performed using this model. In Sec. IV, we discuss the role of Stark shifts in quantum logic gate operations.

\section{MODEL}

Let us consider a three-level atomic configuration (Fig. 1). The atom passes through a bimodal cavity. The modes with annihilation operators $a$ and $b$ interact with the $|e\rangle \leftrightarrow|g\rangle$ and $|e\rangle \leftrightarrow|f\rangle$ transitions, respectively. The Hamiltonian under the rotating-wave approximation can be written as

$$
\begin{aligned}
H= & \hbar\left[\omega_{e g}|e\rangle\left\langle e\left|+\omega_{f g}\right| f\right\rangle\langle f|+\omega_{1} a^{\dagger} a+\omega_{2} b^{\dagger} b\right. \\
& \left.+\left\{g_{1}|e\rangle\left\langle g\left|a+g_{2}\right| e\right\rangle\langle f| b+\text { H.c. }\right\}\right],
\end{aligned}
$$

where $\omega_{l g}(l \in e, f)$ is the atomic transition frequency, $\omega_{i}(i$ $\in 1,2)$ is the frequency of the cavity modes $a$ and $b$, and $g_{i}$ is the atom-cavity coupling constant. We assume $g_{i}$ to be real. The interaction Hamiltonian in the interaction picture can be written as

$$
H=\hbar\left[g_{1}|e\rangle\left\langle g\left|a e^{i \Delta_{1} t}+g_{2}\right| e\right\rangle\langle f| b e^{i \Delta_{2} t}+\text { H.c. }\right],
$$

where $\Delta_{i}=\omega_{e g, f}-\omega_{i}(i \in 1,2)$ is the one-photon detuning of the cavity modes.

If the initial number of photons in the $a$ and $b$ modes are $n$ and $\mu$, respectively, then the state vector of the atom-cavity system can be expanded in terms of the possible basis states in the following way:

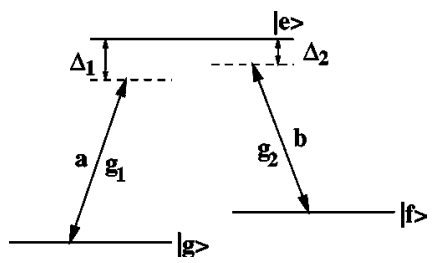

FIG. 1. Three-level atomic configuration with levels $|g\rangle,|f\rangle$, and $|e\rangle$ interacting with two orthogonal modes of the cavity, described by annihilation operators $a$ and $b$. Here $g_{1}$ and $g_{2}$ represent the atom-cavity coupling of the $a$ and $b$ modes with the corresponding transitions, and $\Delta_{i}$ 's $(i \in 1,2)$ are the respective one-photon detunings. 


$$
|\psi(t)\rangle=c_{1}|g\rangle|n, \mu\rangle+c_{2}|e\rangle|n-1, \mu\rangle+c_{3}|f\rangle|n-1, \mu+1\rangle,
$$

where $c_{i}$ 's $(i \in 1,2,3)$ are the probability amplitudes for the corresponding states. The amplitude equations can be obtained from the Schrödinger equation as

$$
\begin{gathered}
\dot{d}_{1}=-i g_{1} \sqrt{n} d_{2}, \\
\dot{d}_{2}=-i\left[g_{1} \sqrt{n} d_{1}+g_{2} \sqrt{\mu+1} d_{3}\right]-i \Delta_{1} d_{2}, \\
\dot{d}_{3}=-i g_{2} \sqrt{\mu+1} d_{2}-i\left(\Delta_{1}-\Delta_{2}\right) d_{3},
\end{gathered}
$$

where the following transformations have been used:

$$
c_{1} \rightarrow d_{1}, \quad c_{2} e^{-i \Delta_{1} t} \rightarrow d_{2}, \quad c_{3} e^{-i\left(\Delta_{1}-\Delta_{2}\right) t} \rightarrow d_{3} .
$$

We now work under the limit of large one-detunings. We assume that $g_{1}=g_{2}=g, \Delta_{i} \gg g$, and $\left(\Delta_{1}-\Delta_{2}\right) \ll g$. In this limit, we put $\dot{d}_{2} \approx 0$ and the amplitude equations (4) reduce to

$$
\begin{gathered}
\dot{d}_{1}=\frac{i g^{2} \sqrt{n}}{\Delta_{1}}\left[\sqrt{n} d_{1}+\sqrt{\mu+1} d_{3}\right], \\
\dot{d}_{3}=-i\left(\Delta_{1}-\Delta_{2}\right) d_{3}+\frac{i g^{2} \sqrt{\mu+1}}{\Delta_{1}}\left[\sqrt{n} d_{1}+\sqrt{\mu+1} d_{3}\right] .
\end{gathered}
$$

We note that Eqs. (5a) and (5b) can be obtained from an effective Hamiltonian given by

$$
\begin{aligned}
H_{\text {eff }}= & -\frac{\hbar g^{2}}{\Delta_{1}}\left[|g\rangle\left\langle g\left|a^{\dagger} a+\right| f\right\rangle\langle f| b^{\dagger} b\right] \\
& -\frac{\hbar g^{2}}{\Delta_{1}}\left[|g\rangle\left\langle f\left|a^{\dagger} b+\right| f\right\rangle\langle g| a b^{\dagger}\right]+\hbar\left(\Delta_{1}-\Delta_{2}\right)|f\rangle\langle f| .
\end{aligned}
$$

Here the first two terms represent the Stark shifts and the next two terms give the interaction leading to a transition from the initial state to the final state. The last term represents a shifting of the level $|f\rangle$ due to the two-photon detuning. From the Hamiltonian (6), one can easily see that the Stark-shift terms are of the same order of magnitude ( $\left.=\hbar g^{2} / \Delta_{1}\right)$ as the coupling term, and thus are as important as the coupling term and should be kept in further discussion. So one cannot ignore these Stark-shift terms from the Hamiltonian. The solution of Eqs. (5a) and (5b) is

$$
\begin{aligned}
d_{1}(t)= & e^{i v t / 2}\left\{\left[\cos \left(\frac{\Omega t}{2}\right)+\frac{i}{\Omega}\left(\Delta_{1}-\Delta_{2}\right) \sin \left(\frac{\Omega t}{2}\right)\right] d_{1}(0)\right. \\
& \left.+\frac{2 i g^{2}}{\Delta_{1} \Omega} \sin \left(\frac{\Omega t}{2}\right) d_{3}(0)\right\},
\end{aligned}
$$

$$
\begin{aligned}
d_{3}(t)= & e^{i v t / 2}\left\{\left[\cos \left(\frac{\Omega t}{2}\right)-\frac{i}{\Omega}\left(\Delta_{1}-\Delta_{2}\right) \sin \left(\frac{\Omega t}{2}\right)\right] d_{3}(0)\right. \\
& \left.+\frac{2 i g^{2}}{\Delta_{1} \Omega} \sin \left(\frac{\Omega t}{2}\right) d_{1}(0)\right\}
\end{aligned}
$$

where

$$
\begin{gathered}
\Omega=\left[\left(\frac{2 g^{2}}{\Delta_{1}}\right)^{2}+\left(\Delta_{1}-\Delta_{2}\right)^{2}\right]^{1 / 2}, \\
\nu=\left[\frac{2 g^{2}}{\Delta_{1}}-\left(\Delta_{1}-\Delta_{2}\right)\right],
\end{gathered}
$$

and we have considered $n=1$ and $\mu=0$. Under the twophoton resonance condition $\Delta_{1}=\Delta_{2}=\Delta$, the solution reduces to

$$
\begin{aligned}
& d_{1}(t)=\frac{1}{2}\left[d_{1}(0)+d_{3}(0)\right]\left(e^{i \theta}-1\right)+d_{1}(0), \\
& d_{3}(t)=\frac{1}{2}\left[d_{1}(0)+d_{3}(0)\right]\left(e^{i \theta}-1\right)+d_{3}(0),
\end{aligned}
$$

where $\theta=2 g^{2} t / \Delta$.

We note that, if we exclude Stark-shift terms from the Hamiltonian (6), and work under two-photon resonance (i.e., $\Delta_{1}=\Delta_{2}=\Delta$ ), then the effective Hamiltonian reduces to

$$
H_{\mathrm{eff}}^{\prime}=-\frac{\hbar g^{2}}{\Delta}\left(S^{-} a^{\dagger} b+S^{+} a b^{\dagger}\right)
$$

where $S^{+}=|f\rangle\langle g|$ and $S^{-}=|g\rangle\langle f|$ are the atomic two-photon creation and annihilation operators, respectively. The solution of the Schrödinger equations using this Hamiltonian is

$$
\begin{aligned}
& d_{1}(t)=\cos \left(\frac{g^{2} t}{\Delta}\right) d_{1}(0)+i \sin \left(\frac{g^{2} t}{\Delta}\right) d_{3}(0), \\
& d_{3}(t)=\cos \left(\frac{g^{2} t}{\Delta}\right) d_{3}(0)+i \sin \left(\frac{g^{2} t}{\Delta}\right) d_{1}(0),
\end{aligned}
$$

which represents a Rabi oscillation of the vector $\left(d_{1}, d_{3}\right)$.

\section{QUANTUM LOGIC GATE OPERATIONS}

In this section, we will show how different kinds of twoqubit logic gates can be performed using the present model.

\section{A. QPG operation}

Let us first consider the solutions of Eqs. (5a) and (5b) under the total effective Hamiltonian (6) (with $\Delta_{1} \neq \Delta_{2}$ ), given by Eqs. (7a) and (7b). From these solutions one can easily see that, if

$$
\frac{\Delta_{1}-\Delta_{2}}{g}=\frac{2}{\left(\Delta_{1} / g\right)},
$$

then, for an interaction time $t=T$ defined by 


$$
g T=\frac{\pi}{\sqrt{2}}\left(\frac{\Delta_{1}}{g}\right)=\frac{\sqrt{2} \pi}{\left(\Delta_{1}-\Delta_{2}\right) / g},
$$

$d_{1}(t)$ becomes -1 for the initial condition $d_{1}(0)=1$. At this particular interaction time, one can perform the following QPG operation:

$$
\begin{gathered}
\left|0_{a}\right\rangle\left|0_{b}, g\right\rangle \rightarrow\left|0_{a}\right\rangle\left|0_{b}, g\right\rangle, \\
\left|0_{a}\right\rangle\left|0_{b}, f\right\rangle \rightarrow\left|0_{a}\right\rangle\left|0_{b}, f\right\rangle, \\
\left|0_{a}\right\rangle\left|1_{b}, g\right\rangle \rightarrow\left|0_{a}\right\rangle\left|1_{b}, g\right\rangle, \\
\left|0_{a}\right\rangle\left|1_{b}, f\right\rangle \rightarrow-\left|0_{a}\right\rangle\left|1_{b}, f\right\rangle,
\end{gathered}
$$

which clearly involves the atomic ground-state basis and the Fock state basis in $b$ mode. It should be mentioned here that we have verified the above analytical results by solving Eqs. (4) numerically for the amplitudes $d_{i}$ also. The numerical results reveal that the adiabatic approximation used in the present problem holds well.

Note that in the above QPG operation we have considered $\Delta_{1} \neq \Delta_{2}$. But if we work under the two-photon resonance condition $\left(\Delta_{1}=\Delta_{2}=\Delta\right)$, then the solutions of Eqs. (5a) and (5b) are given by Eqs. (9a) and (9b). From these solutions, one can easily notice that the time-dependent amplitude of the initial state $\left|0_{a}\right\rangle\left|f, 1_{b}\right\rangle$ is now $\left(e^{2 i g^{2} T / \Delta}+1\right) / 2$ [see Eq. (9b)], which never reaches $(-1)$. Rather for a certain choice of $2 g^{2} T / \Delta(=\pi / 2)$, this becomes $e^{i \pi / 4} / \sqrt{2}$. This clearly shows that the system no longer remains in that state (as is obvious from the factor $1 / \sqrt{2}$ ). A transition takes place to another basis state $\left|1_{a}\right\rangle\left|g, 0_{b}\right\rangle$. In this way, working with the total effective Hamiltonian under two-photon resonance, one cannot perform a phase gate operation. Thus in the present model, the QPG operation can be performed successfully only by avoiding the two-photon resonance condition.

We emphasize that the QPG operation discussed above involves the cavity mode $b$ as well as the ground metastable states $(|g\rangle$ and $|f\rangle)$ of the atom, the transition between which is dipole-forbidden. This is unlike the case in [7], where the authors used two Rydberg states (states with very large quantum numbers) which are dipole-coupled. Thus, the QPG operation discussed in the present paper is not affected by any kind of decoherence due to spontaneous emission of the atomic levels, though it is limited by the cavity lifetime as all operations such as the storage of the photons after initial preparation and detection of the photonic qubit are to be done within the cavity lifetime. To realize the QPG operation against the cavity decay, one has to meet the condition $\pi \Delta_{1} \kappa / \sqrt{2} g^{2}<1$, which directly follows from the condition $T<\kappa^{-1}$ and Eq. (13), where $\kappa$ is the cavity decay constant. A possible parameter zone to satisfy the above condition is $\Delta_{1}=10 \mathrm{~g}$ and $\kappa=0.01 \mathrm{~g}$. This, though challenging for an optical cavity, can be expected to be reached very soon.

Note that if we consider a third atomic metastable state $|k\rangle$ which is an auxiliary state, it is possible to perform the following QPG operation involving the atomic metastable states $(|g\rangle$ and $|k\rangle)$ and the two-mode photonic basis $\left(\left|g_{R}\right\rangle\right.$ $\equiv\left|0_{a}, 1_{b}\right\rangle$ and $\left.\left|e_{R}\right\rangle \equiv\left|1_{a}, 0_{b}\right\rangle\right)$,

$$
\begin{gathered}
|k\rangle\left|g_{R}\right\rangle \rightarrow|k\rangle\left|g_{R}\right\rangle, \\
|k\rangle\left|e_{R}\right\rangle \rightarrow|k\rangle\left|e_{R}\right\rangle, \\
|g\rangle\left|g_{R}\right\rangle \rightarrow|g\rangle\left|g_{R}\right\rangle, \\
|g\rangle\left|e_{R}\right\rangle \rightarrow-|g\rangle\left|e_{R}\right\rangle,
\end{gathered}
$$

at the time defined by Eq. (13). We should mention here that there are several other schemes, which use noninteracting levels to perform logic gates in a two-atom basis. Our model is quite different from other schemes [15-17]. Note that we use a single atom interacting with a two-mode cavity, unlike the cases cited above, which use two atoms interacting with a single-mode cavity.

\section{B. CNOT operation}

A CNOT gate can be implemented from a QPG operation through a rotation of the second qubit before and after the QPG operation. We choose the atom as the second qubit in the present problem. By applying the Hadamard transformation on the atomic state, before and after the QPG operation (14), we obtain the following CNOT operation:

$$
\begin{gathered}
\left|0_{a}\right\rangle\left|0_{b}, g\right\rangle \stackrel{\hat{C}}{\rightarrow}\left|0_{a}\right\rangle\left|0_{b}, g\right\rangle, \\
\left.\left|0_{a}\right\rangle\left|0_{b}, f\right\rangle \rightarrow 0_{a}\right\rangle\left|0_{b}, f\right\rangle, \\
\left|0_{a}\right\rangle\left|1_{b}, g\right\rangle \stackrel{\hat{C}}{\rightarrow}\left|0_{a}\right\rangle\left|1_{b}, f\right\rangle, \\
\quad \hat{C} \\
\left|0_{a}\right\rangle\left|1_{b}, f\right\rangle \rightarrow\left|0_{a}\right\rangle\left|1_{b}, g\right\rangle,
\end{gathered}
$$

where $\hat{C}$ represents the CNOT operation here. Here the Hadamard transformation on the atomic qubit states $|g\rangle$ and $|f\rangle$ can be implemented by applying two resonant $\mathrm{cw}$ fields with equal Rabi frequencies in the respective transitions of the atom. We identify the field qubit as the controlling qubit and the atomic qubit as the controlled qubit. We note that recently DeMarco et al. demonstrated a CNOT gate operation in a single trapped ion interacting with a single Raman pulse [18].

\section{SWAP gate operation}

In order to arrive at SWAP gate, we rewrite the Hamiltonian (6) as an interaction between the two "qubits" in the following way: 


$$
\begin{aligned}
\tilde{H}_{\text {eff }}= & -\frac{\hbar g^{2}}{\Delta_{1}}\left[S^{+} R^{-}+S^{-} R^{+}-2 S^{z} R^{z}+\frac{1}{2}\right] \\
& +\hbar\left(\Delta_{1}-\Delta_{2}\right)\left(S_{z}+\frac{1}{2}\right)
\end{aligned}
$$

where

$$
\begin{gathered}
S^{+}=|f\rangle\left\langle g\left|, \quad S^{-}=\right| g\right\rangle\langle f|, \quad S^{z}=\frac{1}{2}(|f\rangle\langle f|-| g\rangle\langle g|), \\
R^{+}=a^{\dagger} b, R^{-}=a b^{\dagger}, \quad R^{z}=\frac{1}{2}\left(a^{\dagger} a-b^{\dagger} b\right) .
\end{gathered}
$$

Here we identify a single photon in a two-mode cavity as an effective qubit with the two possible states $\left|e_{R}\right\rangle$ and $\left|g_{R}\right\rangle$. The field operator $R$ acts like a spin-1/2 operator in this basis. We now assume that the two-photon resonance condition is satisfied $\left(\Delta_{1}=\Delta_{2}=\Delta\right)$ so that the effective Hamiltonian reads

$$
\tilde{H}_{\mathrm{eff}}=-\frac{2 \hbar g^{2}}{\Delta}\left[S^{x} R^{x}+S^{y} R^{y}-S^{z} R^{z}+\frac{1}{4}\right],
$$

where $S^{x}=\left(S^{+}+S^{-}\right) / 2$ and $S^{y}=\left(S^{+}-S^{-}\right) / 2 i$. This signifies a spin-exchange interaction between two spin-1/2 particles. This kind of interaction is always responsible for swap operation. Defining the unitary operation as

$$
U=\exp \left(-i \tilde{H}_{\mathrm{eff}} \mathrm{t} / \hbar\right)=\exp \left[i \theta\left(S^{x} R^{x}+S^{y} R^{y}-S^{z} R^{z}+\frac{1}{4}\right)\right],
$$

where $\theta=2 g^{2} t / \Delta$, a SWAP gate can be performed for $\theta$ $=\pi\left[U_{\mathrm{SW}}=U(\theta=\pi)\right]$,

$$
\begin{gathered}
|g\rangle\left|g_{R}\right\rangle \stackrel{U_{\mathrm{SW}}}{\rightarrow}|g\rangle\left|g_{R}\right\rangle, \\
|g\rangle\left|e_{R}\right\rangle \stackrel{U_{\mathrm{SW}}}{\rightarrow}-|f\rangle\left|g_{R}\right\rangle, \\
|f\rangle\left|g_{R}\right\rangle \stackrel{U_{\mathrm{SW}}}{\rightarrow}-|g\rangle\left|e_{R}\right\rangle, \\
|f\rangle\left|e_{R}\right\rangle \stackrel{U_{\mathrm{SW}}}{\rightarrow}|f\rangle\left|e_{R}\right\rangle .
\end{gathered}
$$

\section{Role of phases of the coupling constants in a SWAP gate}

In all the above calculations, we have assumed that the field coupling constants $g_{1}$ and $g_{2}$ are equal and in phase. However if they are not so, the general expression for the Hamiltonian [Eq. (6)] under two-photon resonance would be

$$
\begin{aligned}
H^{\prime}= & -\frac{\hbar}{\Delta}\left[\left|g_{1}\right|^{2}|g\rangle\left\langle\left. g\left|a^{\dagger} a+\right| g_{2}\right|^{2} \mid f\right\rangle\left\langle f\left|b^{\dagger} b+g_{1} g_{2}^{*}\right| g\right\rangle\langle f| a^{\dagger} b\right. \\
& \left.+g_{1}^{*} g_{2}|f\rangle\langle g| a b^{\dagger}\right] .
\end{aligned}
$$

Now if we impose the conditions

$$
\left|g_{1}\right|=\left|g_{2}\right|=g, \quad g_{2}=-g_{1},
$$

then the above Hamiltonian can be written as

$$
H^{\prime} \equiv \frac{2 \hbar g^{2}}{\Delta}\left[S^{x} R^{x}+S^{y} R^{y}+S^{z} R^{z}-\frac{1}{4}\right]=\frac{2 \hbar g^{2}}{\Delta}\left[\vec{S} \cdot \vec{R}-\frac{1}{4}\right]
$$

instead of Eq. (19). Then the corresponding unitary operation $U^{\prime}$ becomes

$$
U^{\prime}=e^{-i H^{\prime} t / \hbar}=\exp \left[-i \theta\left(\vec{S} \cdot \vec{R}-\frac{1}{4}\right)\right]=\left[1+\left(e^{-i \theta}-1\right) \hat{P}\right] e^{i \theta} .
$$

Here $\hat{P}=\frac{3}{4}+\vec{S} \cdot \vec{R}$ is the projection operator with the eigenvalues 0 and 1 . The SWAP gate of Sec. III C can also be implemented using the above unitary operator for $\theta=\pi$. It is also very interesting to note that not only for a particular phase relation between $g_{1}$ and $g_{2}$, but for any arbitrary phase between them, the SWAP gate works in the following way:

$$
\begin{gathered}
|g\rangle\left|g_{R}\right\rangle \rightarrow|g\rangle\left|g_{R}\right\rangle, \\
|g\rangle\left|e_{R}\right\rangle \rightarrow-e^{i \phi}|f\rangle\left|g_{R}\right\rangle, \\
|f\rangle\left|g_{R}\right\rangle \rightarrow-e^{-i \phi}|g\rangle\left|e_{R}\right\rangle, \\
|f\rangle\left|e_{R}\right\rangle \rightarrow|f\rangle\left|e_{R}\right\rangle,
\end{gathered}
$$

where $\phi$ is defined through the relation $e^{i \phi}=g_{1} g_{2}^{*} /\left|g_{1}\right|\left|g_{2}\right|$.

We should emphasize that all these universal logic gates are the key resource in quantum computation. Our method and system can also be used to prepare two-particle and three-particle entangled states involving metastable states of the atoms. This can be done by sequentially addressing the atoms by the two-mode cavity under the two-photon resonance condition (cf. $[19,20])$.

\section{ROLE OF STARK SHIFTS IN QUANTUM LOGIC OPERATIONS}

Next we investigate the role of the Stark-shift term in performing the logic gates. Let us consider the Hamiltonian (10) under the two-photon resonance condition which excludes the Stark-shift term. From the corresponding solutions of Eq. (11a) and (11b) for the probability amplitudes, one can obtain the following QPG operation with a $2 \pi$ pulse $\left(2 g^{2} T / \Delta=2 \pi\right)$ :

$$
\begin{gathered}
\left|0_{a}\right\rangle\left|0_{b}, g\right\rangle \rightarrow\left|0_{a}\right\rangle\left|0_{b}, g\right\rangle, \\
\left|0_{a}\right\rangle\left|0_{b}, f\right\rangle \rightarrow\left|0_{a}\right\rangle\left|0_{b}, f\right\rangle, \\
\left|0_{a}\right\rangle\left|1_{b}, g\right\rangle \rightarrow\left|0_{a}\right\rangle\left|1_{b}, g\right\rangle, \\
\left|0_{a}\right\rangle\left|1_{b}, f\right\rangle \rightarrow-\left|0_{a}\right\rangle\left|1_{b}, f\right\rangle .
\end{gathered}
$$

But as soon as we keep the Stark-shift term in the Hamiltonian [see Eq. (6)] and continue to work under the two- 
photon resonance condition, we cannot achieve phase gate operation. It should be borne in mind that one cannot ignore the Stark-shift terms as they are as important as the coupling term in the Hamiltonian. Our Sec. III A shows how to perform the QPG in spite of the Stark-shift term. We used the extra freedom provided by two-photon detunings in our model. We should mention here that many authors have utilized an additional field to cancel the unwanted Stark shifts [21-23]. We also note that in the context of other models, Stark shifts have been used for two-qubit logic [24], the Deutsch-Jozsa algorithm [25], and quantum holography [26].

Note that, recently, Solano et al. reported a QPG operation based on the interaction of a three-level atom in a ladder configuration and two modes of a cavity (each mode can have either zero or one photon). The cavity modes are highly detuned from a single-photon transition (see Fig. 2 of [27]), but are two-photon-resonant. They showed that by excluding the self-energy terms in the effective Hamiltonian, one can perform a QPG operation in photonic Hilbert space $(|0,0\rangle$ $\rightarrow|0,0\rangle,|0,1\rangle \rightarrow|0,1\rangle,|1,0\rangle \rightarrow|1,0\rangle,|1,1\rangle \rightarrow-|1,1\rangle)$.

\section{CONCLUSIONS}

In conclusion, we have presented a system in which a three-level atom in the $\Lambda$ configuration interacts with a high$Q$ bimodal optical cavity, with the cavity modes being highly detuned from the corresponding single-photon transitions. We have shown how a variety of logic operations can be performed using the ground states of the atoms. The associated decoherence due to spontaneous emission is thus negligible, though the quality of the cavity would lead to some decoherence. We further emphasize that Stark shifts are systematically included in our case. Further, the present system can be used to prepare bipartite and tripartite entangled states involving the metastable states of the atoms.
[1] M. A. Nielsen and I. L. Chuang, Quantum Computation and Quantum Information (Cambridge University Press, Cambridge, 2000).

[2] D. P. DiVincenzo, Phys. Rev. A 51, 1015 (1995); Science 270, 255 (1995); S. Lloyd, Phys. Rev. Lett. 75, 346 (1995).

[3] A. Barenco, C. H. Bennett, R. Cleve, D. P. DiVincenzo, N. Margolus, P. Shor, T. Sleator, J. A. Smolin, and H. Weinfurter, Phys. Rev. A 52, 3457 (1995).

[4] J. I. Cirac and P. Zoller, Phys. Rev. Lett. 74, 4091 (1995); Nature (London) 404, 579 (2000).

[5] T. Pellizzari, S. A. Gardiner, J. I. Cirac, and P. Zoller, Phys. Rev. Lett. 75, 3788 (1995).

[6] T. Sleator and H. Weinfurter, Phys. Rev. Lett. 74, 4087 (1995); Q. A. Turchette, C. J. Hood, W. Lange, H. Mabuchi, and H. J. Kimble, ibid. 75, 4710 (1995); X. Maître, E. Hagley, G. Nogues, C. Wunderlich, P. Goy, M. Brune, J. M. Raimond, and S. Haroche, ibid. 79, 769 (1997).

[7] A. Rauschenbeutel, G. Nogues, S. Osnaghi, P. Bertet, M. Brune, J. M. Raimond, and S. Haroche, Phys. Rev. Lett. 83, 5166 (1999).

[8] N. A. Gerschenfeld and I. L. Chuang, Science 275, 350 (1997); B. E. Kane, Nature (London) 393, 133 (1998).

[9] A. Barenco, D. Deutsch, A. Ekert, and R. Jozsa, Phys. Rev. Lett. 74, 4083 (1995).

[10] D. Loss and D. P. DiVincenzo, Phys. Rev. A 57, 120 (1998).

[11] G. K. Brennen, C. M. Caves, P. S. Jessen, and I. H. Deutsch, Phys. Rev. Lett. 82, 1060 (1999); D. Jaksch, H.-J. Briegel, J. I. Cirac, C. W. Gardiner, and P. Zoller, ibid. 82, 1975 (1999); C. Monroe, D. M. Meekhof, B. E. King, W. M. Itano, and D. J. Wineland, ibid. 75, 4714 (1995).

[12] A. Ekert and R. Jozsa, Rev. Mod. Phys. 68, 733 (1996).

[13] A. Kuhn, M. Hennrich, and G. Rempe, Phys. Rev. Lett. 89, 067901 (2002); M. Hennrich, T. Legero, A. Kuhn, and G.
Rempe, ibid. 85, 4872 (2000).

[14] A. S. Parkins, P. Marte, P. Zoller, and H. J. Kimble, Phys. Rev. Lett. 71, 3095 (1993); A. S. Parkins, P. Marte, P. Zoller, O. Carnal, and H. J. Kimble, Phys. Rev. A 51, 1578 (1995).

[15] J. Pachos and H. Walther, Phys. Rev. Lett. 89, 187903 (2002).

[16] E. Jané, M. B. Plenio, and D. Jonathan, Phys. Rev. A 65 , 050302(R) (2002).

[17] X. X. Yi, X. H. Su, and L. You, Phys. Rev. Lett. 90, 097902 (2003); P. Milman, H. Ollivier, F. Yamaguchi, M. Brune, J. M. Raimond, and S. Haroche, J. Mod. Opt. 50, 901 (2003).

[18] B. DeMarco, A. Ben-Kish, D. Leibfried, V. Meyer, M. Rowe, B. M. Jelenković, W. M. Itano, J. Britton, C. Langer, T. Rosenband, and D. J. Wineland, Phys. Rev. Lett. 89, 267901 (2002).

[19] S. Zheng and G.-C. Guo, Phys. Rev. Lett. 85, 2392 (2000); R. G. Unanyan, N. V. Vitanov, and K. Bergmann, ibid. 87, 137902 (2001); X. Zou, K. Pahlke, and W. Mathis, Phys. Rev. A 65, 064303 (2002).

[20] A. Rauschenbeutel, G. Nogues, S. Osnaghi, P. Bertet, M. Brune, J. M. Raimond, and S. Haroche, Science 288, 2024 (2000).

[21] F. Schmidt-Kaler, H. Häffner, S. Gulde, M. Riebe, G. Lancaster, J. Eschner, C. Becher, and R. Blatt, e-print quant-ph/ 0307211.

[22] E. Solano, e-print quant-ph/0310007.

[23] M. Franca Santos, E. Solano, and R. L. de Matos Filho, Phys. Rev. Lett. 87, 093601 (2001).

[24] D. Jonathan and M. B. Plenio, Phys. Rev. Lett. 87, 127901 (2001).

[25] S. Dasgupta, A. Biswas, and G. S. Agarwal (unpublished).

[26] G. S. Agarwal, P. Lougovski, and H. Walther, e-print quant$\mathrm{ph} / 0402116$.

[27] E. Solano, M. Franca Santos, and P. Milman, Phys. Rev. A 64, 024304 (2001). 